\title{
Hollow Platinum Spheres with Nano-Channels: Synthesis and Enhanced Catalysis for Oxygen Reduction
}

\author{
Hao Ming Chen, ${ }^{\dagger}$ Ru-Shi Liu,,${ }^{*} \dagger$ Man-Yin Lo, ${ }^{\ddagger}$ Sung-Chun Chang, ${ }^{\ddagger}$ Li-Duan Tsai,, \\ Yu-Min Peng, and Jyh-Fu Lee ${ }^{\S}$ \\ Department of Chemistry, National Taiwan University, Taipei 106, Taiwan, Materials Chemical Research \\ Laboratories, Industrial Technology Research Institute, Chutung 310, Taiwan, and National Synchrotron \\ Radiation Research Center, Hsinchu 300, Taiwan
}

Received: January 7, 2008

\begin{abstract}
Hollow nanostructures are a particularly interesting class of materials that have unusual chemical and physical characteristics that are determined by their shape and composition. A facile procedure for the hollow $\mathrm{Pt}$ nanosphere catalysts with nanochannels was developed; the catalysts can be simply prepared at room temperature with silver nanoparticles as sacrificial templates. The incomplete shell of hollow nanospheres may provide the interior surface for the catalytic reaction, and the high surface area of Pt nanospheres leads to a higher catalytic activity. Consequently, these results demonstrate a simple route to enhance the catalytic efficiency of Pt catalysts by a simple improvement.
\end{abstract}

\section{Introduction}

Fuel cells have been extensively studied for many decades because of their "clean" energy. ${ }^{1} \mathrm{Pt}$ has stimulated considerable research into its use as a catalyst in the production of hydrogen and particularly in the direct methanol fuel cell (DMFC). ${ }^{2}$ Nevertheless, a critical problem with Pt-based catalysts is their prohibitive cost. There has been an intense search for less expensive, more abundant nonplatinum electrocatalysts that can offer acceptable performance. ${ }^{3}$ To date, efforts have focused on the development of techniques to produce Pt catalysts with a high surface area to achieve high catalytic performance and utilization efficiency. ${ }^{4}$

By processing metal nanostructures into hollow ones, their performance could be further improved because of their relatively lower densities and higher surface areas than their solid counterparts. The fabrication of uniform hollow spheres with nanometer dimensions having tailored structural, optical, and surface properties has been intensively pursued in a wide range of applications. ${ }^{5}$ Hollow nanostructures are a particularly interesting class of materials that have unusual chemical and physical characteristics that are determined by their shape and composition. ${ }^{6}$ For instance, hollow Pd spheres exhibited good catalytic activities in Suzuki cross-coupling reactions and can be reused numerous times without loss of catalytic activity, ${ }^{7}$ whereas solid Pd nanoparticles usually lose their catalytic activities after one cycle of operation. ${ }^{8}$ The galvanic replacement reaction has been exploited as a powerful means of preparing hollow metal nanostructures. ${ }^{6,9}$ Because heterometallic nanoparticles are the resulting product of the galvanic replacement process, some studies on the local structures of these bimetallic

* Author to whom correspondence should be addressed. E-mail: rsliu@ntu.edu.tw.

$\dagger$ National Taiwan University.

* Industrial Technology Research Institute.

$\S$ National Synchrotron Radiation Research Center.
SCHEME 1: Synthetic Routes of Porous Hollow Pt Nanospheres with Ag Cores and Agl-Pt Hollow Nanospheres

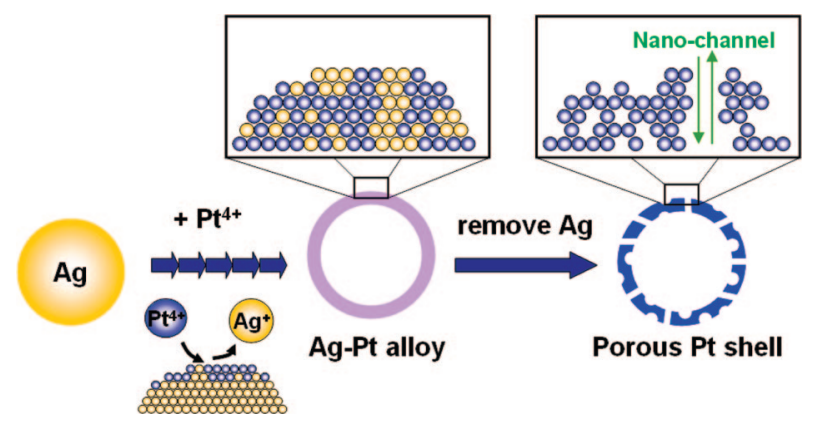

nanoalloys have been demonstrated. ${ }^{10}$ Here, we report on the fabrication of hollow spheres with nanochannels composed of platinum using a modified galvanic replacement reaction and their applications as electrocatalysts. The corresponding stateof-art method used higher temperature for catalyst preparation. ${ }^{5,6}$ However, this replacement approach was operated at room temperature, which made the fabrication of catalysts effortless.

\section{Experimental Section}

Chemicals and Materials. Sodium borohydride (98\%), silver nitrate (99\%), hydrogen hexachloroplatinate (IV) hydrate, and poly(vinyl pyrrolidone) (PVP, $\mathrm{Mw}=58000)$ were obtained from Acros Organics and used without further purification. The water used throughout this investigation was reagent-grade water, produced using a Milli-Q SP ultrapure-water purification system from Nihon Millipore Ltd., Tokyo.

Preparation. Water was used as the solvent in all reactions. For a typical preparation, the silver nanoparticles were prepared by adding $14 \mathrm{~g}$ of PVP to $500 \mathrm{~mL}$ of silver metal salt $\left(\mathrm{AgNO}_{3}\right.$, 

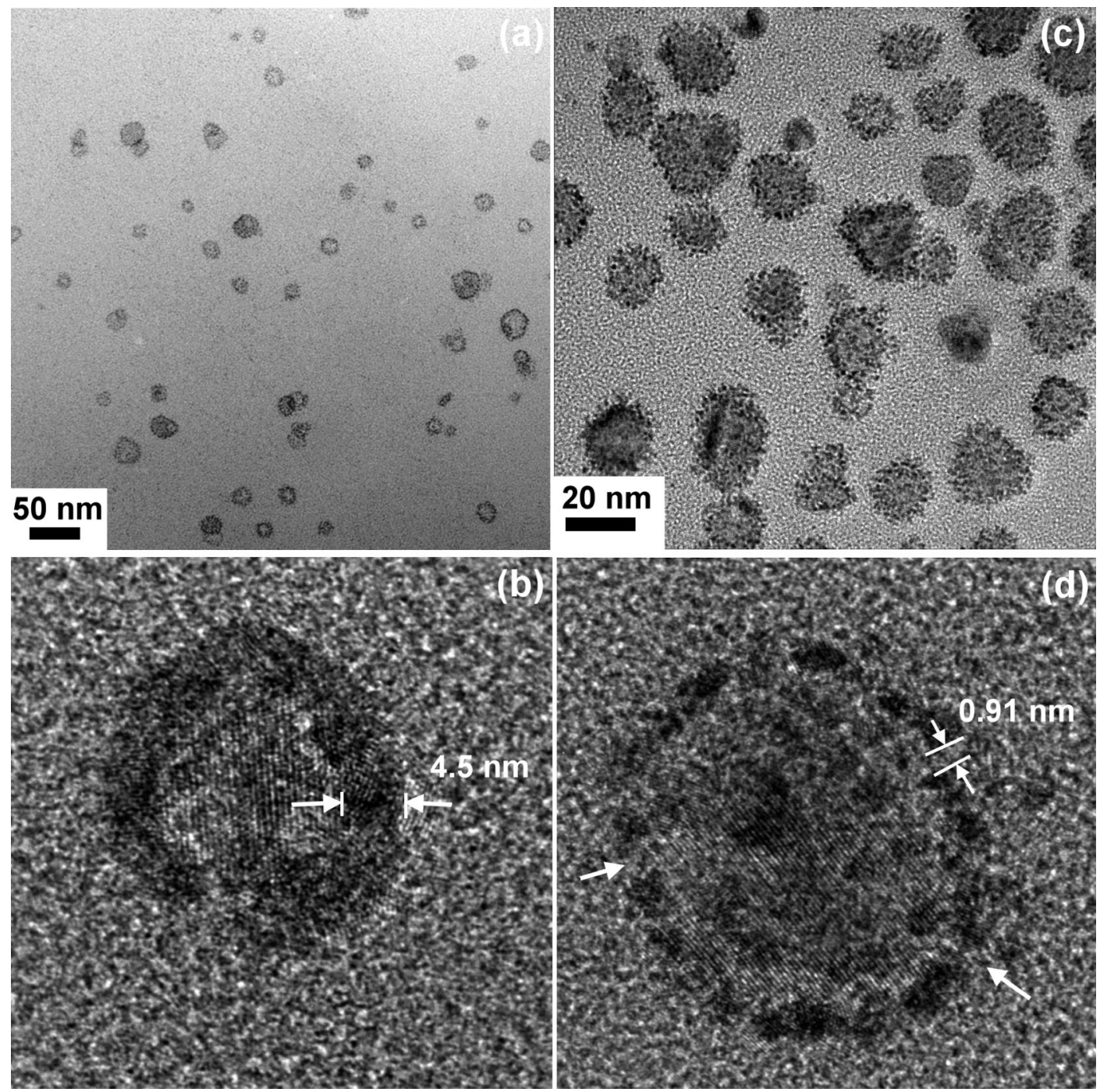

Figure 1. TEM micrographs of the hollow $\mathrm{Ag}-\mathrm{Pt}$ nanoparticles $(\mathrm{a}, \mathrm{b})$ and hollow $\mathrm{Pt}$ shell with nanochannels $(\mathrm{c}, \mathrm{d})$.

$5.6 \mathrm{mM}$ ) solution. The solution was then heated at $60^{\circ} \mathrm{C}$ in an oil bath for $10 \mathrm{~min}$, and then $37 \mathrm{mmol}$ of $\mathrm{NaBH}_{4}$ was added. The mixture was then allowed to react for another $20 \mathrm{~min}$ in an oil bath. The reaction mixture was stirred for $30 \mathrm{~min}$ to remove the excess reducing agent $\left(\mathrm{NaBH}_{4}\right)$. The solution was then allowed to cool before the replacement reaction was performed.

In the replacement experiment, an aliquot of $0.26 \mathrm{M} \mathrm{H}_{2} \mathrm{PtCl}_{6}$ $(10 \mathrm{~mL})$ solution was added dropwise to the silver colloidal solution at room temperature. The mixture continued to undergo the reaction for another $20 \mathrm{~min}$ to yield nanoparticles with $\mathrm{Ag}-\mathrm{Pt}$ alloy shells. In the chemical etching treatment of newly synthesized nanoshells, $50 \mathrm{~mL}(1 \mathrm{M}) \mathrm{NH}_{4} \mathrm{OH}$ solution was added to the as-prepared $\mathrm{Ag}-\mathrm{Pt}$ alloy hollow spheres solution. The reaction mixture was centrifuged at $15000 \mathrm{rpm}$ for $30 \mathrm{~min}$ to remove the $\mathrm{Cl}$ ions. Next, $50 \mathrm{~mL}(1 \mathrm{M}) \mathrm{HNO}_{3}$ was employed to remove silver atoms from the $\mathrm{Ag}-\mathrm{Pt}$ alloy shells. This centrifugation procedure needed to be repeated several times. Vigorous magnetic stirring was maintained throughout the synthesis.

Characterization of Nanoparticles. The UV/vis spectra of the colloidal nanoparticle solutions were obtained using a SHIMADZU UV-1700 spectrophotometer with a $10 \mathrm{~mm}$ quartz cell at room temperature. The surface morphology of the samples was studied using a JEM-2100F (high-resolution transmission electron microscope (HRTEM)) operated at 200 $\mathrm{kV}$ with energy dispersive spectrometer (EDS) analyses. The specimens were obtained by placing many drops of the colloidal solution onto a continuous carbon-coated copper grid and evaporating it in air at room temperature. Prior to specimen preparation, the colloidal solutions were sonicated for $1 \mathrm{~min}$ to improve the dispersion of particles on the copper grid. Preparing an electrode with $\mathrm{Pt} / \mathrm{Pt}$ porous hollow nanoparticles involved dispersing a certain amount of catalysts in water and sonicating it for approximately $30 \mathrm{~min}$ to make a uniform suspension. Then, $20 \mu \mathrm{L}$ of suspension was placed on a glassy carbon disk electrode (5 mm in diameter, PINE: AFE3T050GC) and dried in air at $40{ }^{\circ} \mathrm{C}$. After depositing catalysts, the electrode was covered by $20 \mu \mathrm{L}$ of a $1 \%$ Nafion solution (diluted with isopropyl alcohol). Finally, the electrode was dried in air, and catalyst loading was $2 \mu \mathrm{g} / \mathrm{cm}^{2}$. The activity for oxygen reduction reaction of the catalyst was measured in $\mathrm{O}_{2}$-saturated $0.1 \mathrm{M}$ $\mathrm{HClO}_{4}$ solution obtained using a rotating disk electrode at 2000 rpm. X-ray absorption near-edge spectroscopy (XANES) measurements of the synthesized samples were made using synchrotron radiation at room temperature. Measurements were made at the $\mathrm{Pt}_{3}$-edge (11 $564 \mathrm{eV}$ ) of the sample held at room temperature.

\section{Results and Discussion}

Scheme 1 shows the overall procedure used to synthesize porous Pt hollow spheres. The uniform silver nanospheres were synthesized first. The second step involved the galvanic replace- 

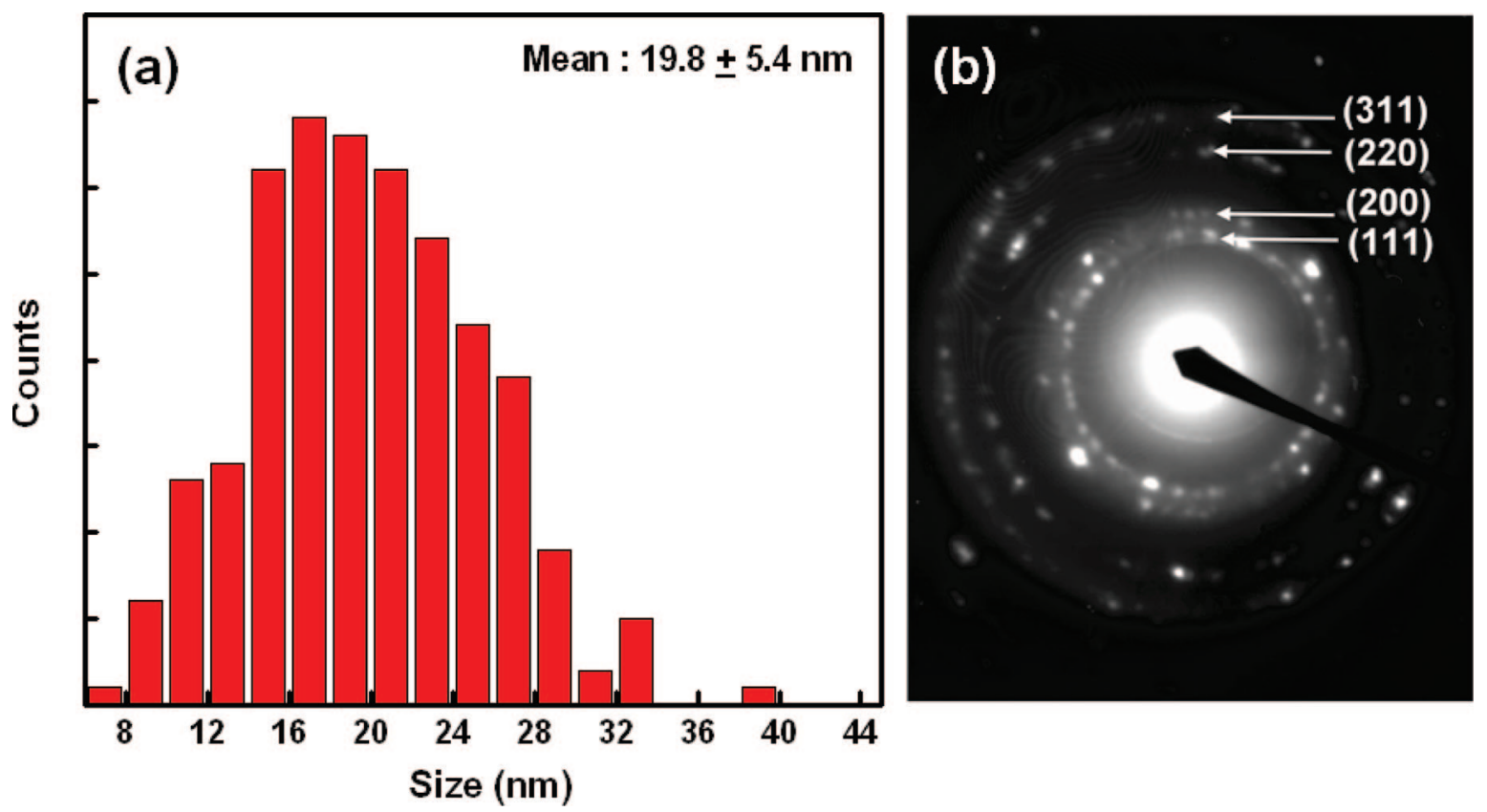

Figure 2. (a) Histograms representing the size distribution of $\mathrm{Ag}-\mathrm{Pt}$ hollow nanospheres. (b) Electron diffraction pattern of porous hollow Pt nanospheres.

ment reaction between $\mathrm{Ag}$ nanoparticles with an aqueous $\mathrm{H}_{2} \mathrm{PtCl}_{6}$ solution at room temperature, whose chemical reaction can be written as $4 \mathrm{Ag}+\mathrm{PtCl}_{6}{ }^{2-} \rightarrow \mathrm{Pt}+4 \mathrm{AgCl}+2 \mathrm{Cl}^{-}$. Pure silver was transformed into a shell made of $\mathrm{Ag}-\mathrm{Pt}$ alloy by galvanic replacement. Since a previous resultb implied that the simultaneous formation of $\mathrm{AgCl}$ might disrupt the epitaxial deposition of gold atoms on the surface of a silver template at room temperature, the presence of $\mathrm{AgCl}$ roughened the surface of the hollow Pt shells, which was manipulated to create a greater surface area for catalytic reaction. The final step involved the removal of the $\mathrm{Ag}$ and $\mathrm{AgCl}$ from the templates by treating the $\mathrm{Ag}-\mathrm{Pt}$ alloy shells with aqueous ammonia and $\mathrm{HNO}_{3}$ to produce nanopores upon the shells of hollow Pt shells. The nanostructures that are involved in each reaction step have been fully characterized using a HRTEM. Figure 1a is a TEM image; it is found that the centers of the spheres are brighter than the edge. $\mathrm{Ag}-\mathrm{Pt}$ alloy nanoparticles as small as $19.8 \mathrm{~nm}$ with a standard deviation of $5.4 \mathrm{~nm}$ were obtained (Figure 2a). The structural details are revealed by HRTEM (Figure 1b). The shells of the Pt hollow nanospheres seem to be smooth and the thickness is $\sim 4.5 \mathrm{~nm}$. The energy dispersive spectromicroscopic analysis indicated that hollow spheres are mainly composed of $\mathrm{Ag}$ and Pt (Supporting Information, Figure S1a). Figure 1c,d presents TEM images of the hollow shells after the removal of $\mathrm{AgCl}$ and $\mathrm{Ag}$. These images clearly revealed that the products were characterized by a porous anostructure (indicated by arrows in Figure 1d) with pore diameters of about $0.91 \mathrm{~nm}$. Notably, the entire structure became segmented owing to the chemical etching. Most interestingly, the thickness of hollow shell after the chemical etching significantly decreased, which implied that the silver atoms were removed from the hollow structures. This feature endows the Pt hollow nanospheres with a high surface area $\left(41 \mathrm{~m}^{2} / \mathrm{g}\right)$. We must emphasize that existence of silver is vital; silver provides active sites for subsequent chemical etching to create nanochannels. EDS elemental analysis revealed that silver and chloride were significantly removed by this treatment (Supporting Information, Figure S1b). The crystalline feature was confirmed by a selected area electron diffraction pattern (see Figure 2b); the Pt hollow nanopheres with nanochannels adopt a typical face-centered-cubic polycrystalline structure.
Figure 3 shows the specific activity for oxygen reduction reaction (ORR) of the commercial Pt catalyst (JM HiSPEC 1000, surface area: $\left.27 \mathrm{~m}^{2} / \mathrm{g}\right)$, hollow Ag-Pt shell $\left(18 \mathrm{~m}^{2} / \mathrm{g}\right)$, and the hollow Pt shell with nanochannels $\left(41 \mathrm{~m}^{2} / \mathrm{g}\right)$. The activity of hollow $\mathrm{Ag}-\mathrm{Pt}$ shell is much lower than that of others owing to the small surface area. The curve of hollow $\mathrm{Pt}$ shell with nanochannels shows a $30 \mathrm{mV}$ shift to more positive potentials compared with commercial Pt. For potentials ranging from 0.9 to $0.8 \mathrm{~V}$, the activity of the hollow Pt shell with nanochannels is higher than that of the commercial catalyst, indicating that the porous hollow shells indeed offer an enhancement in oxygen reduction. The ORR onset potentials are similar for these two surfaces. It is worthwhile to say that higher activity in chemical performance observed here can be attributed to two major factors: (i) nanochannels were formed after the treatment of chemical etching, which might lead to the formation of an activity site in the inner surface of the hollow shell, and (ii) a relatively high density of defects, particularly vacancies were formed at the surface of the shell, which might result in a product with a rough surface rather than a smooth one. The surface roughness could be ascribed to the poor expitaxial growth of $\mathrm{Pt}$ on $\mathrm{Ag}$ as caused by the relatively large difference $(\sim 4.5 \%)$ in lattice constant between $\mathrm{Ag}$ and Pt. These defects (vacancies) with atomic scale and nanochannels were created by removing silver from hollow Ag-Pt alloy; such defects may have high activity as catalysts and provide more bonding sites for chemical reaction. The nanochannels may give rise to the activation of the inner surface and provide a route for the transport of reactant and product.

We could conveniently monitor the replacement between silver spheres and $\mathrm{PtCl}_{6}{ }^{2-}$ solution by spectroscopic means. Figure 4 shows the extinction spectra recorded from aqueous dispersions of silver nanospheres after they had reacted with $\mathrm{Pt}^{4+}$ solution and chemical etching. In this case, the silver nanospheres exhibited a characteristic peak at $\sim 400 \mathrm{~nm}$, which is consistent with previous results. ${ }^{10}$ When a desired volume of $\mathrm{H}_{2} \mathrm{PtCl}_{6}$ solution was added to the dispersion of silver nanoparticles, we observed that an extinction band of silver nanoparticles at $\sim 400 \mathrm{~nm}$ almost damped. This spectral change could be attributed to the formation of thin platinum layers on the 

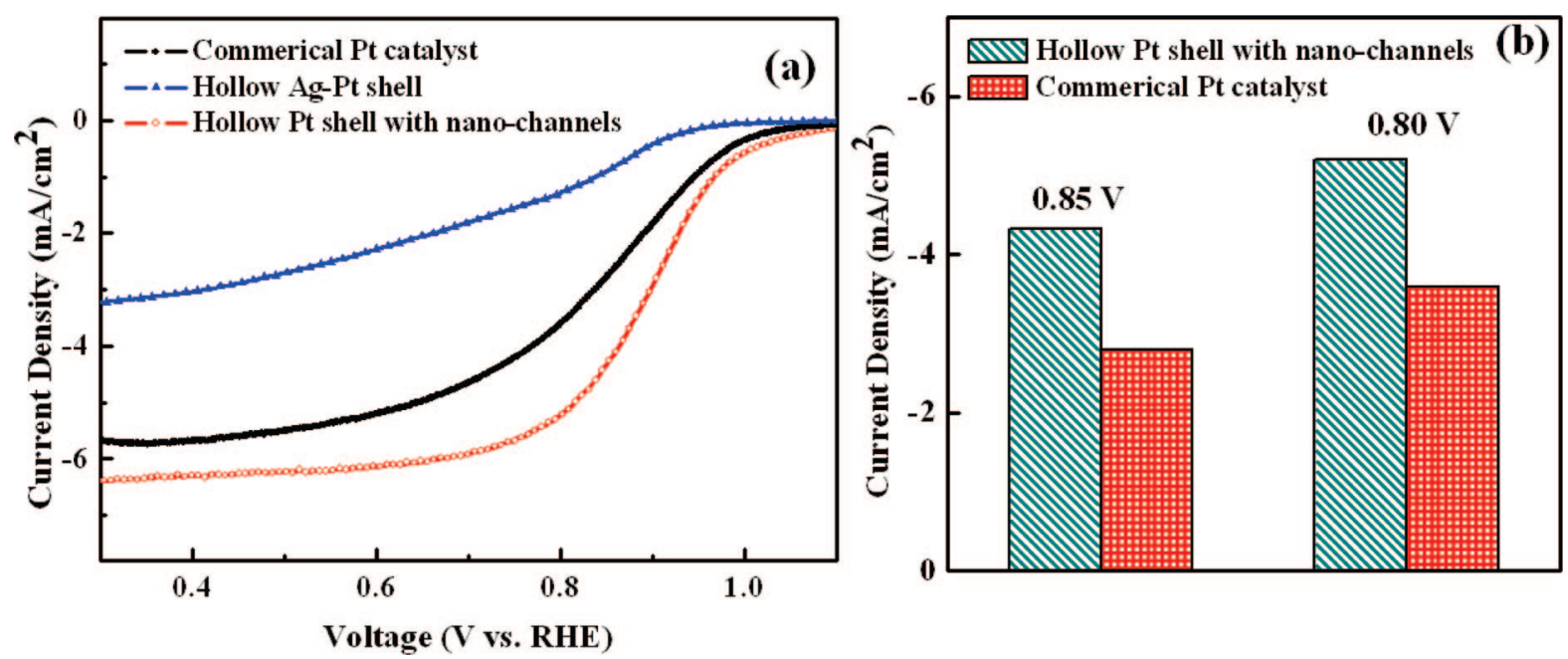

Figure 3. (a) Polarization curve for the ORR on the commercial Pt catalyst, hollow $\mathrm{Ag}-\mathrm{Pt}$ shell, and the hollow Pt shell with nanochannels in 0.1 $\mathrm{M} \mathrm{HClO}_{4}$. (b) Comparison of mass activity for commercial Pt and hollow Pt shell with nanochannels at 0.85 and $0.8 \mathrm{~V}$.

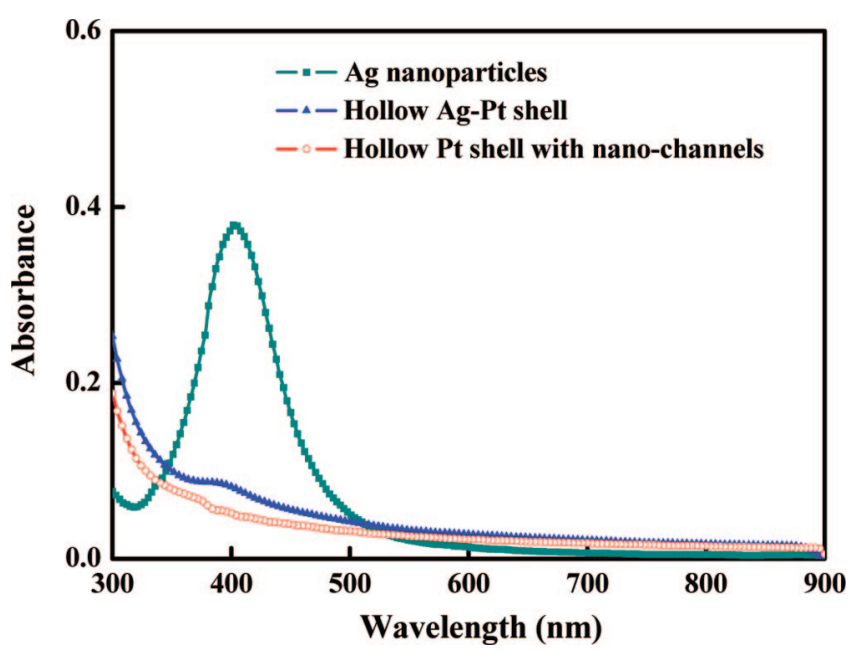

Figure 4. The extinction spectra recorded from aqueous dispersions of silver nanospheres after they had reacted with $\mathrm{Pt}^{4+}$ solution and chemical etching.

surface, which were further transformed into thin shells of $\mathrm{Ag}-\mathrm{Pt}$ alloys. The absorbance slightly decreased after removing of $\mathrm{Ag}$ and/or $\mathrm{AgCl}$.

Earlier work on bimetallic alloy catalysts linked trends in the reactivity to d-band vacancies: the principle explanations for enhanced ORR activity could be attributed to the modification of the electronic structure of Pt (5d orbital vacancies). ${ }^{11}$ To elucidate the $\mathrm{d}$ orbital vacancies of Pt nanoparticles, we determined by XANES the oxidation state of commercial Pt and porous Pt hollow nanospheres. As shown in Figure 5, the area of porous Pt hollow nanospheres $\mathrm{L}_{3}$ threshold resonance line was greater than that of the commercial Pt nanoparticles. The area of the $\mathrm{L}_{3}$ threshold resonance line is proportional to the number of vacant d-electron states, ${ }^{11}$ indicating that the concentration of vacant d-electron states associated with a porous hollow structure is greater than that of a solid one. An increased $5 \mathrm{~d}$ vacancy of $\mathrm{Pt}$ is believed to increase the interaction of $\mathrm{O}_{2}$ and $\mathrm{Pt}$, thereby enhancing the catalytic activity of porous $\mathrm{Pt}$ hollow nanospheres. ${ }^{12}$ The increase of the d-band vacancy may result from the presence of foreign atoms (silver) and/or the atomic scale rough surface of Pt because formation of alloy and structural geometry of Pt have been demonstrated as in the case of d-band vacancy. ${ }^{13}$ Consequently, an enhanced atomic

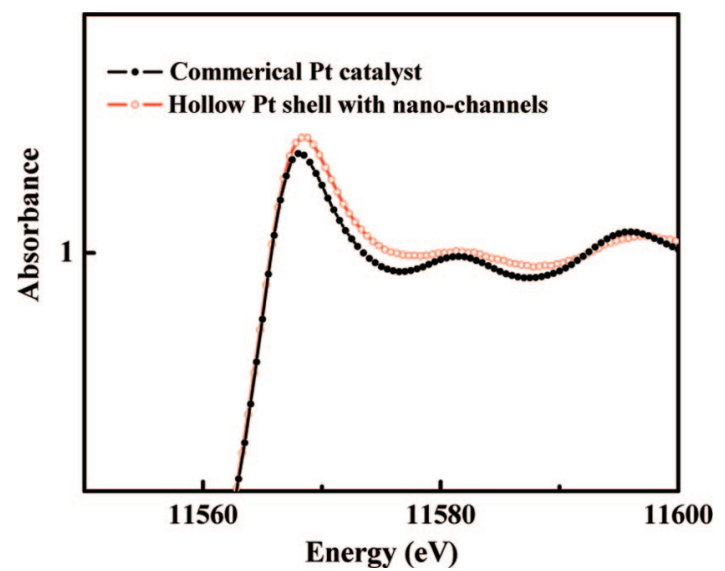

Figure 5. XANES spectra of the commercial Pt and hollow Pt shell with nanochannels at $\mathrm{Pt}_{3}$-edge.

scale surface roughness and low coordination of some atoms may contribute to the observed activity.

\section{Conclusions}

In conclusion, a facile procedure for the synthesis of hollow Pt nanosphere catalysts with nanochannels was developed. The incomplete shell of hollow nanospheres may provide the interior surface for the catalytic reaction, and the high surface area of Pt nanospheres leads to a higher catalytic activity. These metallic hollow nanoparticles could be useful in industrial applications including catalytic nanoreactors and related fields.

Acknowledgment. The authors would like to thank the National Science Council of the Republic of China (contract numbers: NSC 95-ET-7-002-008-ET and NSC 96-2120-M-002019) and the Industrial Technology Research Institute for financially supporting this research.

Supporting Information Available: EDS spectra of $\mathrm{Ag}-\mathrm{Pt}$ hollow nanospheres and porous hollow Pt nanospheres (S1). TEM image of commercial Pt catalyst (S2). This material is available free of charge via the Internet at http://pubs.acs.org.

\section{References and Notes}

(1) (a) Soukharev, V.; Mano, N.; Heller, A. J. Am. Chem. Soc. 2004, 126, 8368. (b) Zhou, S.; Mcllwrath, K.; Jackson, G.; Eichhorn, B. J. Am. Chem. Soc. 2006, 128, 1780. 
(2) (a) Steele, B. C. H.; Heinzel, A. Nature 2001, 414, 345. (b) Bell, A. T. Science 2003, 299, 1688. (c) Rolison, D. R. Science 2003, 299, 1698.

(3) (a) Fernandez, J. L.; Raghuveer, V.; Manthiram, A.; Bard, A. J. J. Am. Chem. Soc. 2005, 127, 13100. (b) Shao, M.-H.; Sasaki, K.; Adzic, R. R. J. Am. Chem. Soc. 2006, 128, 3526.

(4) (a) Zhang, J.; Sasaki, K.; Sutter, E.; Adzic, R. R. Science 2007, 315, 220. (b) Zhang, X.; Chan, K.-Y. Chem. Mater. 2003, 15, 451. (c) Bonnemann, H.; Waldofner, N.; Haubold, H.-G.; Vad, T. Chem. Mater 2002, 14, 1115. (d) Wang, C.; Daimon, H.; Lee, Y.; Kim, J.; Sun, S. J. Am. Chem. Soc. 2007, 129, 6974.

(5) (a) Caruso, F. Adv. Mater. 2001, 13, 11. (b) Sun, Y.; Mayers, B.; Xia, Y. Adv. Mater. 2003, 15, 641. (c) Chen, J.; Saeki, F.; Wiley, B. J.; Cang, H.; Cobb, M. J.; Li, Z.-Y.; Au, L.; Zhang, H.; Kimmey, M. B.; Li, X.; Xia, Y. Nano Lett. 2005, 5, 473. (d) Liang, H.-P.; Zhang, H.-M.; Hu, J.-S.; Guo, Y.-G.; Wan, L.-J.; Bai, C.-L. Angew. Chem., Int. Ed. 2004, 43, 1540. (e) Chen, J.; Wiley, B.; McLellan, J.; Xiong, Y.; Li, Z.-Y.; Xia, Y Nano Lett. 2005, 5, 2058.

(6) (a) Sun, S.; Xia, Y. Nano Lett. 2003, 3, 1569. (b) Sun, S.; Xia, Y. J. Am. Chem. Soc. 2004, 126, 3892. (c) Sun, S.; Wiley, B.; Li, Z.-Y.; Xia, Y. J. Am. Chem. Soc. 2004, 126, 9399. (d) Liang, H.-P.; Zhang, H.-M.; Hu, J.-S.; Guo, Y.-G.; Wan, L.-J.; Bai, C.-L. Angew. Chem., Int. Ed. 2004 43, 1540. (e) Yang, J.; Lee, J. Y.; Too, H.-P.; Valiyaveettil, S. J. Phys. Chem. B 2006, 110, 125.

(7) Kim, S.-W.; Kim, M.; Lee, W. Y.; Hyeon, T. J. Am. Chem. Soc. 2002, 124, 7642 .
(8) (a) Sun, Y.; Xia, Y. Science 2002, 298, 2176. (b) Sun, Y.; Tao, Z.; Chen, J.; Herricks, T.; Xia, Y. J. Am. Chem. Soc. 2004, 126, 5940. (c) Li, Y.; Hong, X. M.; Collard, D. M.; El-Sayed, M. A. Org. Lett. 2000, 2, 2385.

(9) (a) Chen, H. M.; Liu, R.-S.; Asakura, K.; Lee, J.-F.; Jang, L.-Y.; Hu, S.-F. J. Phys. Chem. B 2006, 110, 19162. (b) Chen, H. M.; Chia, F. H.; Liu, R.-S.; Asakura, K.; Lee, J.-F.; Jang, L.-Y. J. Phys. Chem. C 2007, 111, 5909. (c) Chen, H. M.; Liu, R.-S.; Asakura, K.; Jang, L.-Y.; Lee, J.-F. J. Phys. Chem. C 2007, 111, 18550. (d) Lee, W.-R.; Kim, M. G.; Choi, J.-R.; Park, J.-I.; Ko, S. J.; Oh, S. J.; Cheon, J. J. Am. Chem. Soc. 2005, $127,16090$.

(10) Mallin, M. P.; Murphy, C. J. Nano Lett. 2002, 2, 1235.

(11) (a) Mukerjee, S.; Srinivasan, S.; Soriaga, M. P. J. Electrochem. Soc. 1995, 142, 1409. (b) Lytle, F. W.; Wei, P. S. P.; Greegor, R. B.; Via, G. H.; Sinfelt, J. H. J. Chem. Phys. 1979, 70, 4849. (c) Mansour, A. N.; Cook, J. W., Jr.; Sayers, D. E. J. Phys. Chem. 1984, 88, 2330.

(12) (a) Toda, T.; Igarashi, H.; Watanabe, M. J. Electrochem. Soc. 1998, 145, 4185. (b) Zhang, J.; Mo, Y.; Vukmirovic, M. B.; Klie, R.; Sasaki, K.; Adzic, R. R. J. Phys. Chem. B 2004, 108, 10955.

(13) Ankudinov, A. L.; Rehr, J. J.; Low, J. J.; Bare, S. R. J. Chem. Phys. 2002, 116, 1911.

JP8017698 